Rev. Int. Contam. Ambie. 36 (4) 945-956, 2020

https://doi.org/10.20937/RICA.53855

\title{
UTILIZACIÓN DE UN BIORREACTOR CON MEMBRANAS SUMERGIDAS PARA EL TRATAMIENTO DE AGUAS RESIDUALES PROCEDENTES DE UN HOSPITAL ONCOLÓGICO
}

Utilization of a submerged membrane bioreactor for the treatment of wastewater from an oncological hospital

\author{
Yusmel GONZÁLEZ-HERNÁNDEZ ${ }^{1 *}$, Isariebel QUESADA PEÑATE², Sylvie SCHETRITE², \\ Ulises Javier JÁUREGUI-HAZA ${ }^{1,3}$ y Claire ALBASI ${ }^{2}$
}

${ }^{1}$ Instituto Superior de Tecnologías y Ciencias Aplicadas, Universidad de La Habana (InSTEC-UH), Quinta de los Molinos, Av. Salvador Allende 1110, e/ Infanta y Boyeros, Plaza de la Revolución, La Habana, Cuba, AP 6163.

${ }^{2}$ Université de Toulouse, Laboratoire de Génie Chimique, UMR 5503, BP 84234, Campus INP-ENSIACET, 4 allée Emile Monso, 31030 Toulouse Cedex 4, France.

${ }^{3}$ Instituto Tecnológico de Santo Domingo (INTEC), Av. de Los Próceres 49, Los Jardines del Norte 10602 , Santo Domingo, República Dominicana, AP 342-9 y 249-2.

*Autor para correspondencia: yusmel.gonzalez.hernandez@gmail.com

(Recibido: enero de 2020; aceptado: mayo de 2020)

Palabras clave: lodos activados, adaptación, aguas residuales hospitalarias, compuestos orgánicos persistentes.

\section{RESUMEN}

La contaminación de aguas superficiales con los denominados contaminantes emergentes (fármacos y pesticidas, entre otros) constituye uno de los grandes problemas ambientales del siglo XXI. Aunque los sistemas convencionales de lodos activados no son capaces por sí solos de llevar a cabo la descontaminación de estas aguas, recientemente la tecnología de biorreactores con membranas sumergidas (BMS) ha sido considerada para el tratamiento de estas aguas contaminadas, ya que los lodos activados que forman parte de dichos sistemas trabajan en condiciones de operación no convencionales (alta edad del lodo y alta concentración de biomasa). Esto puede favorecer tanto el crecimiento paulatino del consorcio bacteriano como el desarrollo de capacidades fisiológicas y de adaptación de los microorganismos para degradar un compuesto orgánico específico. En el presente trabajo se realizó el seguimiento del funcionamiento de un BMS instalado a la salida de un hospital oncológico, para lo cual se determinaron la demanda química de oxígeno y el nitrógeno en el residual de entrada, en el sobrenadante y en el permeado del BMS. Finalmente se estimó el coeficiente de rendimiento heterótrofo mediante pruebas respirométricas, observándose el fenómeno de almacenamiento de sustrato como respuesta de los microorganismos para el desarrollo de capacidades fisiológicas de adaptación. En general, los resultados obtenidos muestran la capacidad de adaptación de los microorganismos a la presencia de fármacos procedentes de un hospital oncológico, así como la eficiencia de estos sistemas para el tratamiento de aguas residuales hospitalarias.

Key words: activated sludge, adaptation, hospital wastewater, organic persistent compounds. 


\begin{abstract}
Surface waters pollution with the so-called emerging pollutants (pharmaceutical compounds and pesticides, among others) is one of the great environmental problems of the 21 st century. Even though conventional activated sludge systems alone are not capable of carrying out the decontamination of these waters, the technology of submerged membrane bioreactors (SMBR) has been recently considered for the treatment of these contaminated waters, since the activated sludges that are part of these systems work under non-conventional operating conditions (high sludge age and high biomass concentration). This can favor the gradual growth of the bacterial community, as well as the development of physiological and adaptive capacities of microorganisms to degrade a specific organic compound. In the present work, the operation of a pilot SMBR installed at the exit of an oncology hospital was monitored, for which the chemical oxygen demand and nitrogen in the residual input, in the supernatant and in the permeate of the SMBR were determined. Finally, the heterotrophic yield coefficient was estimated by means of respirometric tests, observing the substrate storage phenomenon as a response of microorganisms for the development of physiological adaptive capacities. In general, the results obtained show the ability of microorganisms to adapt to the presence of pharmaceutical compounds from an oncology hospital, as well as the efficiency of these systems for the hospital wastewater treatment.
\end{abstract}

\section{INTRODUCCIÓN}

Los biorreactores con membranas (BRM) se han convertido en una tecnología ampliamente utilizada como tratamiento avanzado de aguas residuales urbanas e industriales (Ferrero et al. 2012, Kumar et al. 2014, Yeole et al. 2018). Los BRM son sistemas en los que se integra la degradación biológica de los efluentes residuales con la filtración con membranas (Cicek et al. 1999). Desde hace muchos años estos sistemas han mostrado su eficiencia en el tratamiento de aguas residuales municipales e industriales (Jiménez et al. 2010, Yeole et al. 2018). En las últimas dos décadas las tecnologías de BRM han crecido exponencialmente debido a las ventajas que ofrecen con relación a los procesos convencionales de tratamiento de aguas residuales, a saber: menor impacto ambiental, mejor calidad de los efluentes y mejor control de los procesos (Song et al. 2007, Zarragoitia-González et al. 2009a, Buer y Cumin 2010, Lin et al. 2011, Hoinkis et al. 2012, Keskes et al. 2012, Judd 2016, Roccaro y Vagliasindi 2020). En los últimos años, el empleo de BRM para el tratamiento de aguas residuales contaminadas con fármacos se ha incrementado considerablemente (Tambosi et al. 2010, Rudin et al. 2018). La efectividad de los BRM para la remoción de microcontaminantes está directamente relacionada con la capacidad de estos sistemas para operar en condiciones no convencionales (alta edad del lodo y alta concentración de biomasa, entre otras). La operación de estos sistemas a altas edades de lodo puede favorecer el crecimiento paulatino de bacterias y la diversidad del consorcio bacteriano, así como el desarrollo de capacidades fisiológicas y de adaptación de las bacterias para degradar un compuesto orgánico específico (Aubenneau et al. 2010, Sipma et al. 2010). Hay algunos estudios en los cuales se reporta el empleo de estas tecnologías para el tratamiento de aguas residuales hospitalarias, donde se establece que los microorganismos no sólo logran adaptarse a los fármacos, sino que incluso llegan a degradarlos (Radjenović et al. 2009, Aubenneau et al. 2010, Sipma et al. 2010, Tambosi et al. 2010, Nguyen et al. 2017, 2019, Svojitka et al. 2017, Ouarda et al. 2018, Tran et al. 2019).

La mayor ventaja potencial de esta tecnología está en el campo de la reutilización del agua. Esto se debe a que las membranas de los reactores pueden ser de ultrafiltración, por lo que retienen las bacterias, algunos virus y muchos componentes orgánicos e inorgánicos que frecuentemente se encuentran en los efluentes de los tratamientos biológicos convencionales (de Luca et al. 2013). Por tanto, dependiendo del uso que se le quiera dar, el efluente de un BMS puede ser adecuado para la reutilización directa en la agricultura, la industria o el uso doméstico de agua no potable (Zanetti et al. 2010). Estas son algunas de las razones por las cuales la investigación en el campo de los BMS está actualmente en continuo crecimiento dado el interés comercial y científico que han despertado. Hasta la fecha no son muchos los trabajos que han reportado la capacidad de adaptación de los lodos de un BRM a fármacos (Radjenović et al. 2009, Delgado et al. 2010, Tambosi et al. 2010, Shen et al. 2014, Nguyen et al. 2019, Tran et al. 2019). 
En los últimos años se ha publicado un número considerable de trabajos relacionados con el empleo de biorreactores con membranas para el tratamiento de aguas residuales. A pesar de que sólo una fracción de estas publicaciones corresponde al empleo de dichas tecnologías para el tratamiento de aguas residuales hospitalarias, su número se ha incrementado a partir de 2005. Esto puede corroborarse en la figura 1, donde se muestran los resultados de una búsqueda bibliográfica realizada en el portal Science Direct hasta 2019 empleando las palabras clave MBR and wastewater, MBR and hospital wastewater, MBR and industrial wastewater.

Teniendo en cuenta la importancia del tratamiento de aguas residuales hospitalarias y la reserva existente en cuanto al empleo de la tecnología de los BMS, en el presente trabajo se evaluó el funcionamiento de un BMS piloto instalado a la salida de un hospital oncológico, a partir de la determinación de parámetros físicos y químicos tales como sólidos suspendidos totales (SST), demanda química de oxígeno (DQO) y $\mathrm{N}$ en el residual de entrada, en el sobrenadante y en el permeado del BMS. Finalmente, se evaluó la actividad de la biomasa heterótrofa durante la degradación de la materia carbonada presente en el agua residual hospitalaria, empleando pruebas respirométricas.

\section{MATERIALES Y MÉTODOS}

\section{Descripción de la instalación}

En el presente estudio se trabajó con aguas residuales hospitalarias en condiciones reales (una descripción más detallada del contenido de fármacos del agua residual hospitalaria en estudio se puede encontrar en Quesada et al. 2015), para lo cual se empleó un BMS piloto (Fig. 2), instalado a la salida del hospital oncológico de Purpan, Toulouse, Francia, el cual fue inoculado con lodo activado procedente de una estación municipal de tratamiento (BRM, Nailloux, Francia). El BMS piloto, completamente automatizado, estaba acoplado a una computadora, lo que permitía su control y operación a distancia. La instalación está formada por un reactor biológico de $1000 \mathrm{~L}$ de volumen útil y de $1100 \mathrm{~L}$ de volumen total, operando con $488 \mathrm{~L}$ de lodos.

En el reactor biológico se delimitan dos zonas fundamentales: una zona aerobia, en la que tienen lugar la degradación del carbono orgánico por las bacterias heterótrofas y la nitrificación por parte de las bacterias autótrofas, y una zona anóxica donde ocurren los procesos de desnitrificación, llevados a cabo fundamentalmente por bacterias heterótrofas en condiciones anóxicas, aunque recientemente se han encontrado algunas bacterias que logran desnitrificar en condiciones aerobias (Gerardi 2002). El lodo se agita de manera permanente para lograr la homogeneidad de la mezcla y garantizar una mejor distribución del sustrato; de esta manera se alcanza un mejor contacto bacteria-sustrato. La zona aerobia se aireó periódicamente por un flujo de burbujas finas. Estos ciclos de aireación se modificaron en el transcurso del estudio, disminuyendo su duración y aumentando la de los periodos sin aireación (60-45 min de aireación/60-150 min sin aireación), lo cual permitió alcanzar un equilibrio entre los procesos aerobios y anóxicos.

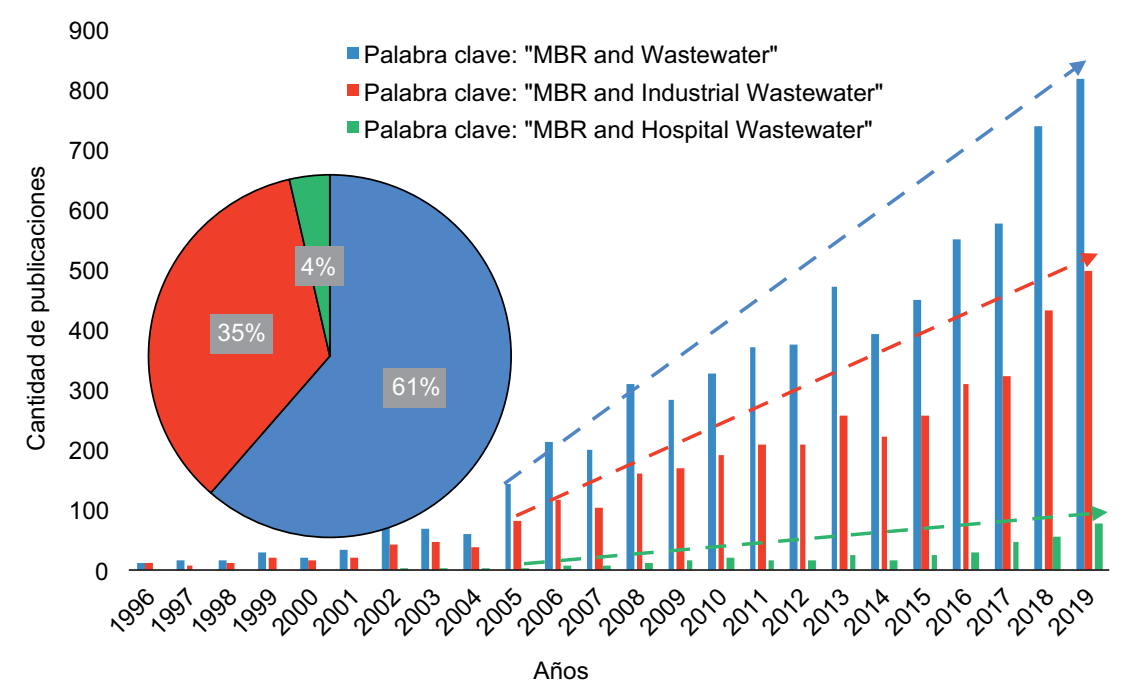

Fig. 1. Número de publicaciones anuales relacionadas con el empleo de los biorreactores con membranas. 


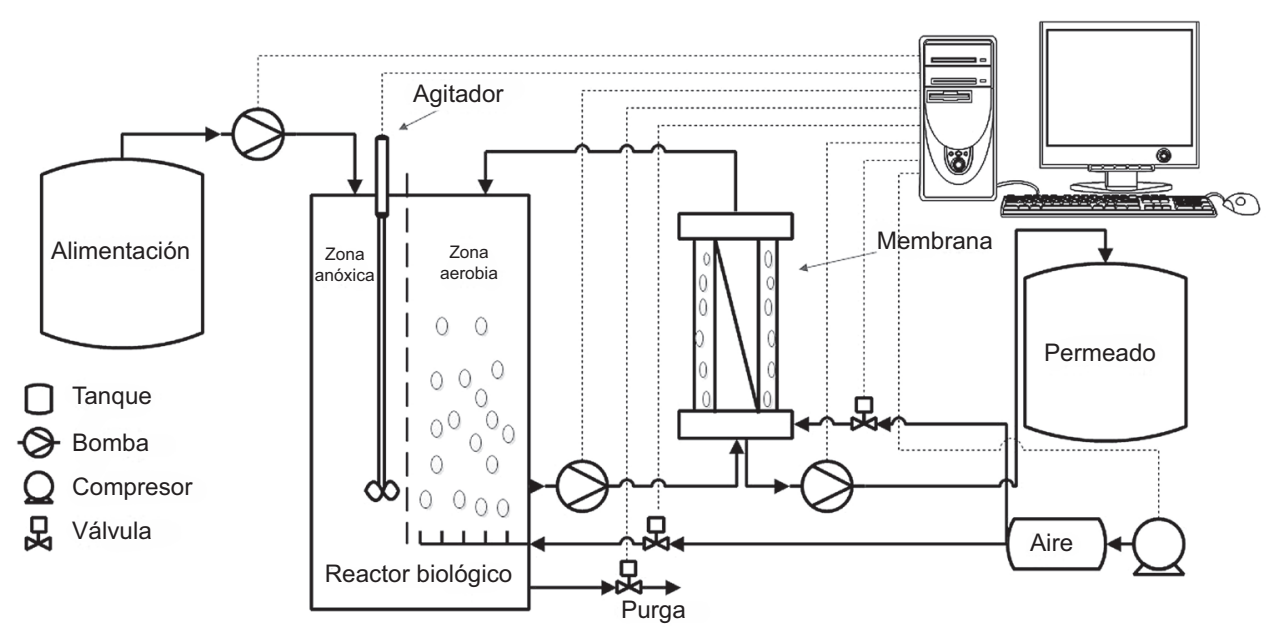

Fig. 2. Esquema general del BMS piloto.

La purga del sistema se realiza de tal forma que se garantiza una edad del lodo de 40 días. La instalación posee, además, un módulo de membranas poliméricas de fibras huecas $(0.2 \mu \mathrm{m})$ suministrada por POLYMEM (polisulfona, $7.5 \mathrm{~m}^{2}$ ), en el cual se lleva a cabo el proceso de separación física del agua tratada de la mezcla de lodo mediante la filtración. A diferencia de otras instalaciones, las membranas se encuentran sumergidas en un módulo que se encuentra fuera del reactor biológico, por el cual se hacen recircular $200 \mathrm{~L}$ de lodo por hora. Este diseño posibilita una mejor manipulación del módulo de membranas para la realización de la limpieza química una vez que se alcanza el valor de presión transmembranal crítica ( 0.35 bar) o el recambio cuando se cumple su tiempo de vida útil.

La filtración a través de la membrana se realizó de forma cíclica ( 2 min de filtración/2 min de relajación). Como resultado de la filtración, se deposita cierta cantidad de materia particulada, coloidal o soluble sobre la superficie de la membrana o en el interior de sus poros. Esto provoca un aumento de la presión transmembranal en el tiempo, ya que el flujo instantáneo de filtración se mantiene constante a lo largo de la operación del sistema a razón de 37 L por hora. Por ello en la parte inferior del módulo de membranas se encuentra un difusor, por el cual se inyectan pulsos de burbujas gruesas intermitentes (1 min aireación/1 min sin aireación) que al ascender chocan con las paredes de la membrana generando fuerzas de cizallamiento sobre su superficie, logrando desprender cierta cantidad de lodo.

El tiempo de retención hidráulico fue de 26 h. Durante todo el estudio se monitorearon los principales parámetros del sistema biológico y de filtración. Para el caso del sistema biológico el monitoreo se realizó mediante el empleo de un muestreador automático.

\section{Caracterización del residual de entrada, del lodo y del efluente del BMS a escala piloto}

La caracterización del residual de entrada constituye generalmente una tarea muy laboriosa, pero al mismo tiempo indispensable cuando se quiere estudiar la eficiencia de un sistema de tratamiento de aguas residuales. En el caso del BMS piloto de Purpan, se conformaron muestras compuestas semanales tanto para el agua residual (alimentación) como para el efluente del BMS. Para la preparación de dichas muestras se tomaron volúmenes de $30 \mathrm{~mL}$ de agua residual cada 15 min durante un periodo de 7 días (de lunes a domingo), empleando un muestreador automático. Al trabajar con esta muestra media semanal se logra una mejor aproximación a la realidad, mientras que una muestra puntual podría conducir a resultados inexactos.

Por otra parte, teniendo en cuenta que la composición de los lodos es más estable y que su edad para la caracterización es de 40 días, se tomaron dos muestras puntuales por semana. Tanto al residual de entrada como a los lodos y al permeado se les realizaron análisis de SST, DQO y N, cuyos resultados se muestran en el cuadro I.

Para la determinación de la DQO y el N se emplearon los métodos espectrofotométricos estandarizados de la firma Hach, mientras que los SST se determinaron por el método descrito en la norma NF T 90-105 (AFNOR 1978). 
CUADRO I. ANÁLISIS REALIZADOS AL AGUA RESIDUAL, LOS LODOS Y AL EFLUENTE DEL BMS.

\begin{tabular}{|c|c|c|c|c|c|c|}
\hline Muestra & $\begin{array}{l}\text { SST } \\
(\mathrm{g} / \mathrm{L})\end{array}$ & $\begin{array}{c}\text { DQO } \\
(\mathrm{mg} / \mathrm{L})\end{array}$ & $\begin{array}{c}\text { N-Total } \\
(\mathrm{mg} \mathrm{N} / \mathrm{L})\end{array}$ & $\begin{array}{c}\mathrm{N}^{-\mathrm{NH}_{4}}{ }^{+} \\
(\mathrm{mg} \mathrm{N} / \mathrm{L})\end{array}$ & $\begin{array}{c}\mathrm{N}^{-\mathrm{NO}_{3}^{-}} \\
(\mathrm{mg} \mathrm{N} / \mathrm{L})\end{array}$ & 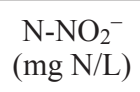 \\
\hline Agua residual & $x$ & $x$ & $x$ & $x$ & $x$ & $x$ \\
\hline Lodos & $x$ & - & - & - & - & - \\
\hline Sobrenadante & - & $x$ & - & - & - & - \\
\hline Permeado & - & $x$ & $x$ & $x$ & $x$ & $x$ \\
\hline
\end{tabular}

\section{Estudios de respirometría Respirómetro}

En este estudio se empleó un respirómetro discontinuo (batch) en que el aporte de oxígeno depende de dos valores de consigna, un máximo y un mínimo de concentración de oxígeno dentro del recipiente donde se lleva a cabo la respirometría (Fig. 3). Se inyecta aire hasta alcanzar el valor máximo de concentración de oxígeno establecido. Una vez alcanzado este valor se deja de inyectar oxígeno y se espera a que los microorganismos lo consuman hasta llegar a la consigna mínima; entonces se vuelve a airear. Se mide el tiempo que emplean los microorganismos para consumir el oxígeno, con lo que se obtiene la velocidad instantánea de consumo de oxígeno. El respirómetro está formado por un recipiente de cristal de $2 \mathrm{~L}$ con un agitador magnético cuya función es lograr la homogenización de la mezcla de lodo activado. Durante los experimentos la temperatura del sistema se mantuvo constante a $25^{\circ} \mathrm{C}$ por medio de un baño termostático.

El oxígeno disuelto se midió en el reactor por medio de un oxímetro (YSI-51) conectado a una computadora que disponía como interfase una tarjeta de adquisición de datos (IOtech). Los datos de la concentración de oxígeno se almacenaron a intervalos de $1 \mathrm{~s}$ utilizando un programa desarrollado sobre el sistema informático Dasylab v9.0.

\section{Coeficiente de rendimiento heterótrofo}

El coeficiente de rendimiento heterótrofo constituye un parámetro estequiométrico que define la cantidad de biomasa heterótrofa que se produce a partir de la cantidad de sustrato fácilmente biodegradable consumido. A partir de la realización de pruebas de respirometría y mediante la adición de una cantidad de sustrato fácilmente biodegradable se puede determinar el coeficiente de rendimiento heterótrofo. Para ello se mide la cantidad de oxígeno que se consume para la degradación biológica de una cantidad conocida de sustrato fácilmente biodegradable y se parte de la premisa de que una parte del sustrato añadido se utiliza directamente para la producción de biomasa, mientras que la otra parte se emplea para la producción de la energía que se utiliza en los procesos de transporte y el mantenimiento de la célula. Esta energía se produce a partir de la respiración (Fig. 4).

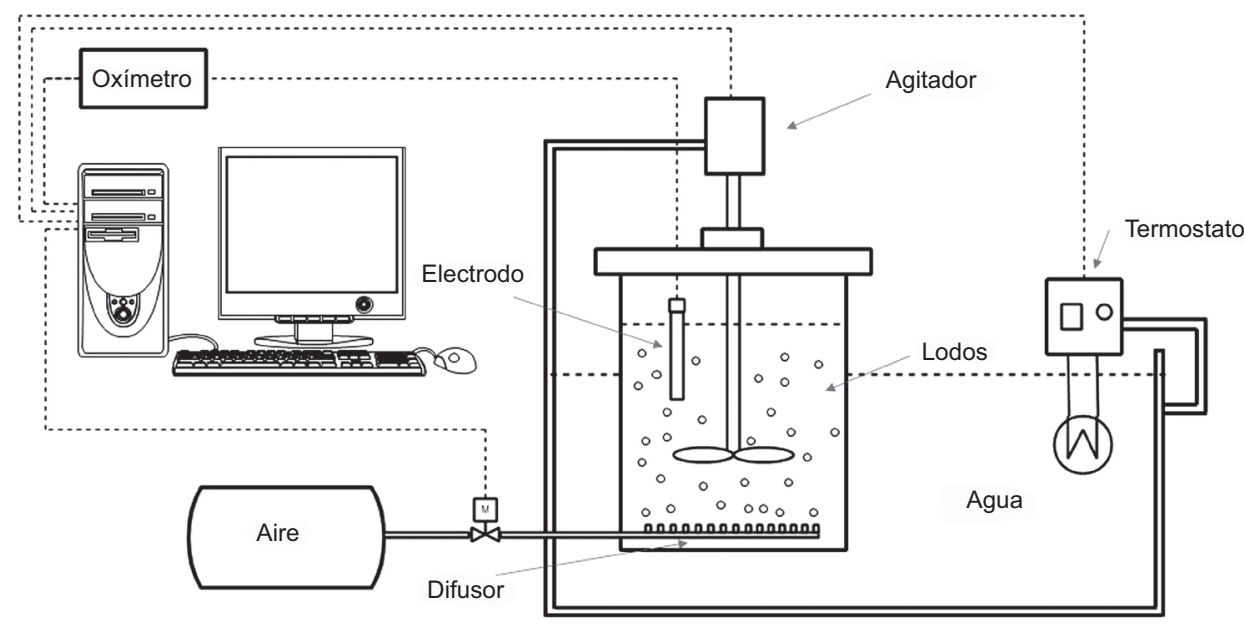

Fig. 3. Esquema general del respirómetro. 


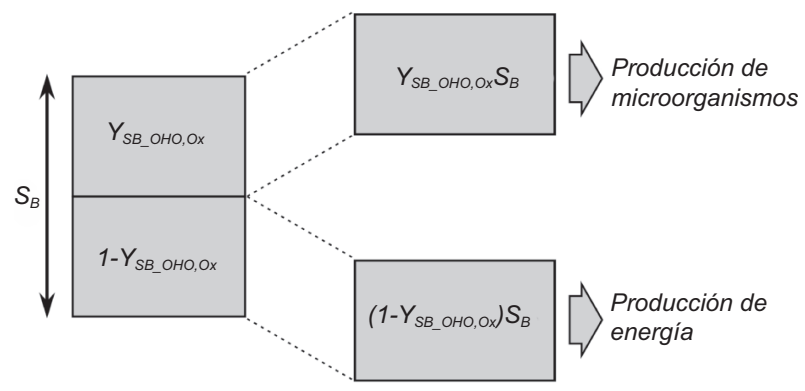

Fig. 4. Relación entre el coeficiente de rendimiento heterótrofo y el consumo de sustrato fácilmente biodegradable.

Para la estimación del coeficiente de rendimiento heterótrofo $\left(\mathrm{Y}_{\mathrm{SB} \_\mathrm{OHO}, \mathrm{Ox}}\right)$ se llevaron a cabo varias pruebas respirométricas en que, a partir de la condición endógena del lodo activado, se adicionó una cantidad conocida de sustrato soluble fácilmente biodegradable $\left(\mathrm{CH}_{3} \mathrm{COONa}\right)$ y se registró el aumento de la velocidad de consumo de oxígeno (VCO) hasta alcanzar los niveles iniciales de respiración endógena, lo que representa el final de la degradación del sustrato alimentado. En cada prueba respirométrica se agregaron $15 \mathrm{mg}$ de alliotiurea (ATU) antes de añadir el sustrato, para inhibir la actividad de las bacterias nitrificantes. El oxígeno consumido para la degradación del sustrato añadido puede determinarse como el área debajo de la curva que está delimitada por una recta que parte de la línea base de la respiración endógena antes del pulso respirométrico hasta la línea base correspondiente a la respiración endógena después de la degradación del sustrato añadido (Fig. 5).

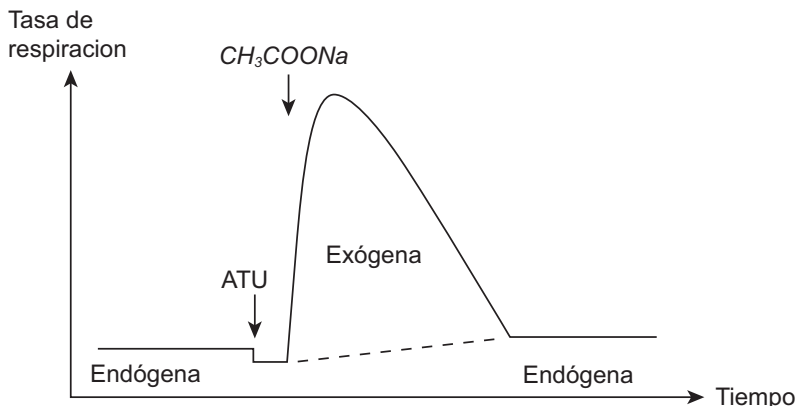

Fig. 5. Protocolo de respirometría para la determinación del coeficiente de rendimiento heterótrofo.

Por tanto, si se grafica el oxígeno consumido para cada cantidad de sustrato añadido se obtiene una recta cuya pendiente corresponde a 1-Y $\mathrm{Y}_{\mathrm{SB} \_\mathrm{OHO}, \mathrm{Ox}}$ según la ecuación 1.

Oxígeno consumido $=\left(1-\mathrm{Y}_{S B \_O H O, O x}\right) S_{\mathrm{B}}$

\section{RESULTADOS Y DISCUSIÓN}

\section{Caracterización del residual de entrada y de los lodos \\ Sólidos suspendidos totales}

Conocer la magnitud de los sólidos suspendidos totales que vienen con la alimentación es de vital importancia, pues gran parte de ellos son biodegradables, otra parte es inerte y una pequeña porción corresponde a la biomasa, por lo que pasa a formar parte de los lodos, lo que dificulta en ocasiones determinar la verdadera fracción de biomasa en el biorreactor. En este trabajo se determinó la concentración de SST dentro del biorreactor y los provenientes de la alimentación. En la figura 6 se muestra el comportamiento de la concentración media semanal de los SST en la alimentación y en el biorreactor.

En general, la concentración de SST en la alimentación varía muy poco en el tiempo, a diferencia de lo que ocurre en el interior del BMS, donde se aprecian diferentes comportamientos en el tiempo. Al inicio se muestra un comportamiento estable hasta los 36 días, etapa que corresponde a la arrancada y puesta en marcha de la instalación, seguido por un descenso brusco como resultado de problemas con la programación de la purga de lodos, y algunos problemas presentados con la alimentación. Una vez corregida esta falla, se pudo observar cómo con el transcurso del tiempo se produce un aumento en la concentración de sólidos suspendidos en el interior del reactor. McArdell et al. (2011) estudiaron la eficiencia de un BMS piloto en la remoción de aguas residuales hospitalarias operando con una edad de lodo de alrededor de 30-50 días. Este sistema alcanzó una concentración de lodo de $2 \mathrm{~g} / \mathrm{L}$, valor inferior a los valores registrados por el BMS piloto en estudio, que fueron superiores a 4 $\mathrm{g} / \mathrm{L}$ durante casi todo el periodo de experimentación, operando con una edad de lodo de 40 días.

\section{Demanda química de oxígeno}

En la figura 7 se muestra el seguimiento del comportamiento de la media semanal de la demanda química de oxígeno (DQO) del residual de entrada, del sobrenadante y del permeado, así como la eficiencia de remoción del sistema.

La DQO de alimentación se mantiene estable en el transcurso del tiempo, con una media semanal de alrededor de $500 \mathrm{mg} \mathrm{DQO} / \mathrm{L}$, mientras que el permeado y el sobrenadante presentan valores muy cercanos, aunque el valor de la DQO del permeado es más bajo que el del sobrenadante. Esto se debe fundamentalmente al paso del líquido a través de la torta durante la filtración, donde una pequeña parte 


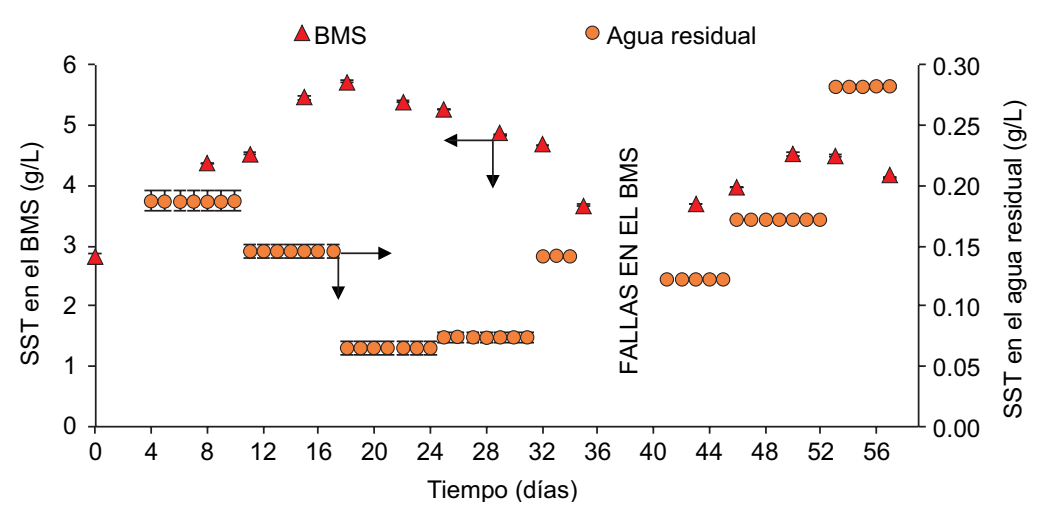

Fig. 6. Comportamiento de los sólidos suspendidos totales en la alimentación y en el interior del BMS piloto.

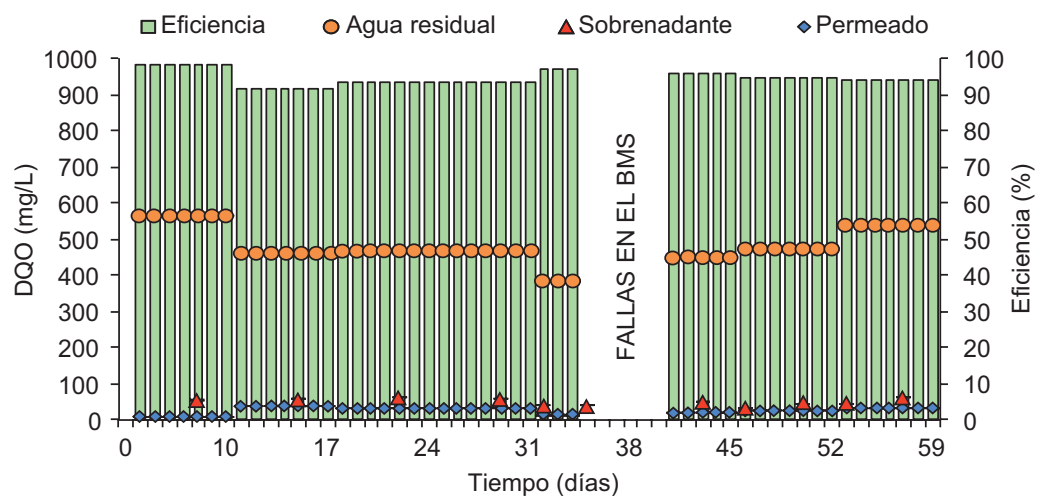

Fig. 7. Comportamiento y eficiencia de remoción de la DQO en el BMS piloto.

de la DQO queda retenida o es adsorbida por la torta de lodo formada sobre la superficie de la membrana (Ersahin et al. 2016). Los valores de DQO del permeado se mantienen por debajo de $40 \mathrm{mg} \mathrm{DQO} / \mathrm{L}$ durante todo el periodo de experimentación, valor que cumple con la norma de vertimiento de aguas residuales establecida por la Comunidad Europea en la directiva 91/271/CEE del 21 de mayo de 1991 $(<125 \mathrm{mg}$ DQO/L) (CEE 1991).

En la figura 7 se puede observar además que el sistema en estudio, alimentado con un residual hospitalario, logra eliminar hasta un $96 \%$ de la DQO de partida en el sistema. Braak (2012) trabajó con un BRM piloto alimentado con aguas residuales municipales con una eficiencia de remoción de DQO del $96 \%$. Delgado et al. (2010) evaluaron la influencia de la ciclofosfamida y de sus principales metabolitos en el comportamiento de la biomasa en un BRM con una eficiencia de remoción de la DQO global del sistema superior al $90 \%$. Zarragoitia-González (2009b) trabajó con un BMS a escala de laboratorio, alimentado con aguas residuales municipales que alcanzó una eficiencia de remoción de la DQO del $88 \%$, valor que también es inferior al obtenido en el caso en estudio. Di Bella et al. (2013) estudiaron el funcionamiento de un BRM piloto, el cual mostró una alta eficiencia de remoción cercana al $97 \%$. Nguyen et al. (2017) emplearon dos biorreactores con membranas esponjosas de fibra hueca y plana, en el tratamiento de 16 aguas residuales hospitalarias, logrando una eficiencia de remoción de la DQO para ambos BRM del $96 \%$ al $97 \%$. En general, en comparación con las eficiencias de remoción de DQO reportadas por otros autores, la observada en el sistema en estudio es superior a pesar de que en este caso la alimentación contiene productos farmacéuticos, lo cual demuestra que los microorganismos son capaces de adaptarse a la presencia de fármacos.

\section{Nitrógeno}

En la figura 8 se puede observar que, con el paso del tiempo, el nitrógeno en forma de nitrato y de nitrito va disminuyendo en el permeado. Este comportamiento es el resultado de la disminución del tiempo 


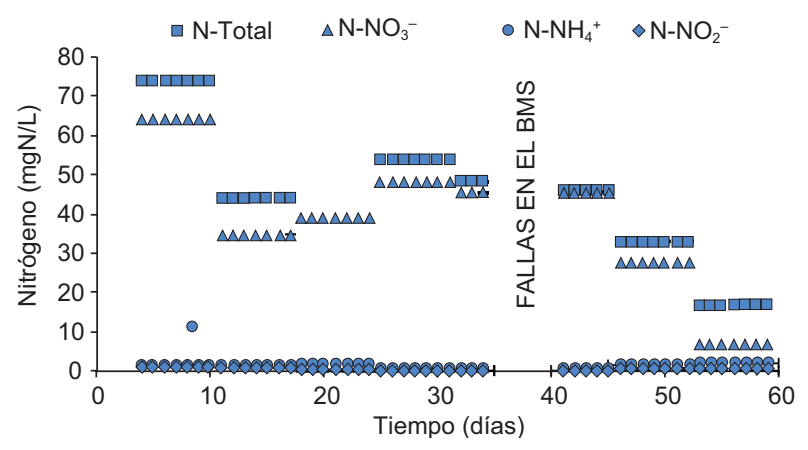

Fig. 8. Comportamiento del nitrógeno en el permeado del BMS piloto.

de aireación con burbujas finas y del incremento del tiempo sin aireación hasta que se obtienen niveles de nitrato y nitrito permisibles en el permeado de acuerdo con las normas de vertimiento establecidas.

La concentración de nitrito en el BMS constituye un parámetro crucial en el funcionamiento del sistema biológico, ya que un incremento en la concentración de nitrito puede inhibir los procesos de nitrificación y por tanto llevar el sistema a un mal funcionamiento (Gerardi 2002).

Durante todo el periodo de experimentación el $\mathrm{pH}$ del sistema se mantuvo entre 7 y 8 mediante la adición de bicarbonato de calcio. La necesidad de alcalinizar el sistema se debe fundamentalmente a que el consumo de alcalinidad en el proceso de nitrificación es mayor que la producción de alcalinidad durante los procesos de desnitrificación. Si se establecen relaciones estequiométricas se obtiene que, por cada miligramo de amonio nitrificado, se consumen
$7.14 \mathrm{mg}$ de alcalinidad $\left(\mathrm{CaCO}_{3}\right)$, mientras que por cada miligramo de nitrato desnitrificado se generan $3.57 \mathrm{mg}$ de alcalinidad $\left(\mathrm{CaCO}_{3}\right)$, lo cual representa la mitad de lo que se consume durante la nitrificación (Gerardi 2002).

En la figura 8 puede observarse la similitud entre el comportamiento del nitrógeno total en la alimentación y en el permeado. La diferencia estriba sólo en las magnitudes, siendo mayor en el caso de la alimentación, y se basa fundamentalmente en la cantidad de nitrógeno orgánico que no logra pasar a través de la membrana más el nitrógeno que escapa del sistema en forma de dinitrógeno como resultado de los procesos de desnitrificación.

En la figura 9 puede observarse que la eficiencia de remoción de nitrógeno aumenta con el tiempo, alcanzando un valor hasta del $80 \%$. Este fenómeno puede atribuirse a la intensificación de los procesos de desnitrificación, como resultado de la disminución de los tiempos de aireación con burbujas finas y el aumento de los tiempos de parada de aireación. En el caso en estudio existieron dificultades para establecer los mejores tiempos de aireación y parada de aireación con burbujas finas, debido a que mediante la recirculación del lodo a través del módulo membranal se incorpora oxígeno al sistema, como resultado de la aireación con burbujas gruesas. En general, a partir de la modificación de la duración de los periodos aerobios/anóxicos se lograron valores de $\mathrm{N}$ en el permeado de $17 \mathrm{mg} \mathrm{N} / \mathrm{L}$, ligeramente superiores a la norma de vertimiento de aguas residuales establecida por la Comunidad Europea en la directiva 91/271/CEE del 21 de mayo de 1991 (< $15 \mathrm{mg} \mathrm{N} / \mathrm{L}$ ) (CEE 1991). Cuando se analiza la composición del

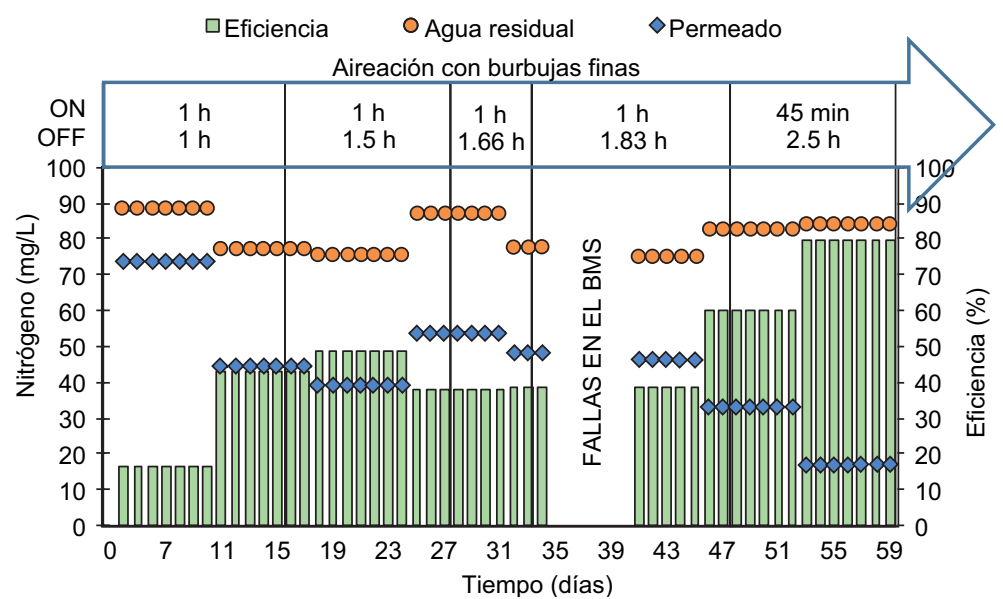

Fig. 9. Comportamiento y eficiencia de remoción del nitrógeno total en el BMS piloto. 
nitrógeno total en el permeado, el $41 \%$ corresponde a nitrógeno en forma de nitrato. Esto significa que incrementando aún más la duración de los periodos anóxicos se podría favorecer el proceso de desnitrificación para lograr niveles de nitrógeno inferiores a los establecidos en la norma de vertimiento de aguas residuales de la Comunidad Europea.

McArdell et al. (2011) estudiaron el desempeño de un BMS piloto en la remoción de aguas residuales hospitalarias obteniendo una buena eficiencia de remoción del nitrógeno de $85-90 \%$, valores superiores a los del BMS en estudio, debido fundamentalmente a que en el BMS de dichos autoresla zona anóxica estaba separada de la zona aerobia, lo cual permitía una mejor operación del sistema, de manera que se garantizaban condiciones favorables para la desnitrificación. Por el contrario, en el BMS piloto de este estudio las condiciones anóxicas y aerobias se garantizan en el mismo tanque, lo cual dificulta establecer condiciones favorables para que ambos sistemas trabajen al máximo de sus potencialidades. Por su parte, Nguyen et al. (2017) obtuvieron una eficiencia de remoción del nitrógeno en el tratamiento de aguas residuales hospitalarias que fluctuó entre el 85 y el $96 \%$.

\section{Coeficiente de rendimiento heterótrofo}

Con el propósito de obtener el valor más aproximado de $\mathrm{Y}_{\mathrm{SB} \_ \text {OHO,Ox }}$ se realizaron varias pruebas respirométricas en las que varió la concentración del acetato de sodio. Para ello se trabajó con una disolución madre de acetato de sodio a $7 \mathrm{~g} / \mathrm{L}$. En este caso se realizó una réplica del primer experimento, obteniéndose un error de reproducibilidad de $\pm 4.5 \%$, por lo que no fue necesario realizar más réplicas para los experimentos restantes. Cuando se grafica el consumo de oxígeno para cada cantidad de sustrato añadida en cada experimento se obtiene una recta cuya pendiente equivale a $1-Y_{\text {SB_OHO,Ox }}$ (Fig. 10).

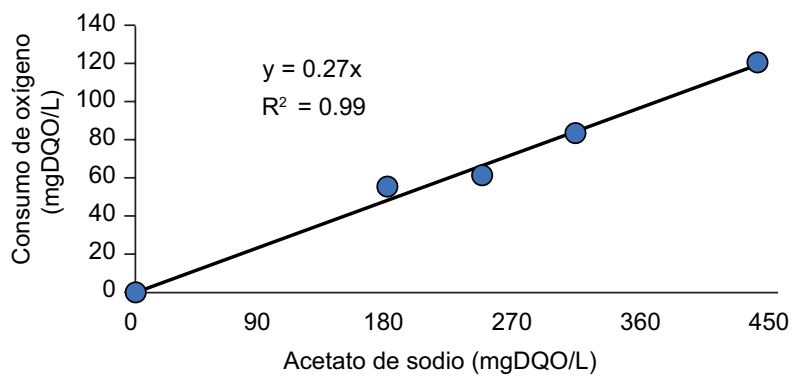

Fig. 10. Determinación del coeficiente de rendimiento heterótrofo del lodo del BMS piloto.

A partir de la figura 10 puede determinarse el coeficiente de rendimiento heterótrofo, que en este caso es de 0.73 g DQO $\mathrm{XOHO}_{\mathrm{gDQO}} \mathrm{gB}_{\mathrm{SB}}$ El valor obtenido es superior al reportado por Henze (2000) (0.63 g DQO Хоно/gDQO ${ }_{\mathrm{SB}}$ ) y al reportado por Vivekanandan y Rao (2017) recientemente. Sin embargo, también se han reportado valores superiores en otros estudios (e.g., Strotmann et al. 1999).

El coeficiente de rendimiento heterótrofo es un parámetro estequiométrico que permite determinar la cantidad de biomasa producida a partir de la cantidad de sustrato que se consumide. En este caso, el hecho de haber obtenido un valor mayor al reportado inicialmente por Henze (2000) puede explicarse por dos aspectos fundamentales: 1) que este coeficiente se estima experimentalmente en un sistema biológico donde existe una gran diversidad de microorganismos, y la composición del consorcio de microorganismos depende de las condiciones de alimentación y del medio en el cual se desarrollan, y 2) el fenómeno de almacenamiento de sustrato por la biomasa, una capacidad que desarrollan los microorganismos en sistemas que presentan irregularidades en su alimentación, la cual ocasiona que en los periodos de alimentación acumulen sustrato en forma de polímeros como reserva para los largos periodos sin alimentación. En la figura 11 se muestra

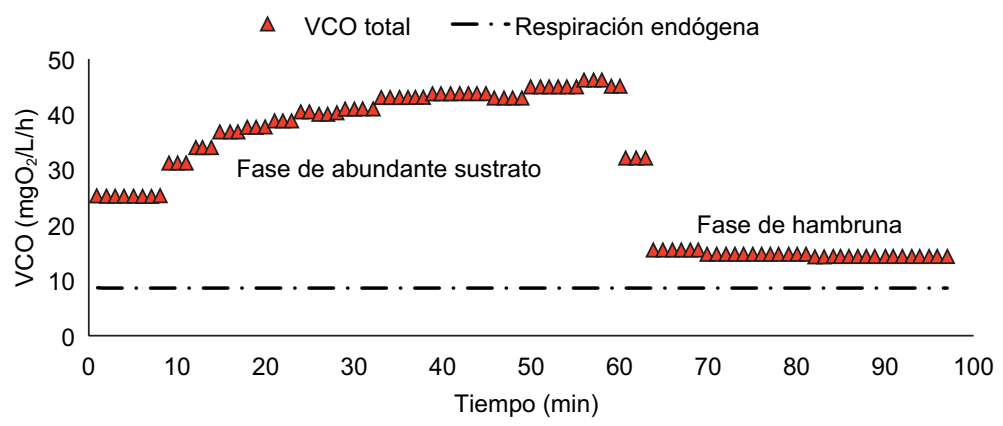

Fig. 11. Perfil respirométrico correspondiente a la degradación de 103 mgDQO/L de acetato de sodio. 
el perfil respirométrico correspondiente a la degradación de $103 \mathrm{mg} \mathrm{DQO} / \mathrm{L}$ de acetato de sodio, donde se corrobora lo antes planteado.

Cuando se analizan los respirogramas correspondientes a la determinación de este coeficiente estequiométrico se pueden observar dos etapas fundamentales: una primera etapa donde se produce una respuesta respirométrica rápida que corresponde al almacenamiento de una parte del sustrato añadido y a la utilización de la otra parte en el crecimiento de la biomasa, y una segunda etapa en la cual se observa una respuesta respirométrica menos rápida que corresponde a la utilización del sustrato almacenado una vez que se ha consumido todo el sustrato fácilmente biodegradable añadido (Carta et al. 2001, Beun et al. 2002).

En general se han obtenido resultados similares a los reportados para sistemas que tratan aguas residuales urbanas, en los cuales ocurre el fenómeno de almacenamiento de sustrato. Por otro lado, aun cuando en este estudio no existen irregularidades con la alimentación, se observa el fenómeno de almacenamiento de sustrato, lo cual puede atribuirse a que la irregularidad de la alimentación está relacionada fundamentalmente con la carga de fármacos y la baja disponibilidad de materia carbonada biodegradable en el residual de entrada. Este fenómeno de almacenamiento de sustrato podría ser una respuesta de los microorganismos para desarrollar capacidades fisiológicas y adaptarse a la presencia de los fármacos, e incluso degradarlos.

El valor del coeficiente de rendimiento heterótrofo obtenido no corresponde al valor real del mismo. En este caso se obtuvo una sobrestimación de dicho coeficiente debido a que el método respirométrico convencional no es adecuado para los sistemas en que ocurre el fenómeno de almacenamiento de sustrato. Esta técnica respirométrica falla debido a que asume que la degradación de todo el sustrato fácilmente añadido al inicio de la prueba respirométrica corresponde al oxígeno medido, cuando en realidad corresponde con una menor cantidad de sustrato fácilmente biodegradable debido a las transformaciones que éste sufre en un sistema en el cual tiene lugar el fenómeno de almacenamiento (González-Hernández et al. 2019).

\section{CONCLUSIONES}

A partir del seguimiento realizado al BMS piloto se pudo constatar que los lodos activados empleados en este estudio lograron adaptarse a la presencia de fármacos, alcanzando una eficiencia en la remoción de la DQO y del nitrógeno de hasta un 96 y un
$80 \%$, respectivamente. Estos resultados se consideran aceptables para un sistema de tratamiento de aguas residuales procedentes de un hospital oncológico en el que se desecha una gran variedad de fármacos, ya que se logró obtener un permeado con una DQO que cumple con la norma de vertimiento de aguas residuales establecida por la Comunidad Europea, en tanto que para el nitrógeno se obtuvo un valor ligeramente superior al establecido en dicha norma, lo que podría resolverse mediante un incremento en la duración de los periodos anóxicos. El rendimiento de eliminación de la DQO es similar al reportado por otros autores, mientras que la eliminación de nitrógeno es ligeramente inferior a la obtenida en BMS que trabajan con aguas residuales municipales y hospitalarias. Se estimó el coeficiente de rendimiento heterótrofo, obteniéndose un valor de $0.73 \mathrm{~g} \mathrm{DQO} \mathrm{XOHO}_{\mathrm{g}} \mathrm{DQO}_{\mathrm{SB}}$, similar a los valores reportados para sistemas que tratan aguas residuales urbanas en los que ocurre el fenómeno de almacenamiento de sustrato. En este caso, el fenómeno de almacenamiento de sustrato podría atribuirse a una respuesta de los microorganismos para desarrollar capacidades fisiológicas con el fin de adaptarse a la presencia de los fármacos e incluso para degradarlos. Los resultados obtenidos demuestran la utilidad de estas tecnologías en el tratamiento de aguas residuales contaminadas con fármacos.

\section{AGRADECIMIENTOS}

Este trabajo se realizó en el marco del proyecto ANR PANACEE con la cooperación de la dirección del Hospital Universitario de Purpan, Toulouse, Francia y el apoyo de la Sección de Cooperación de la Embajada de Francia en Cuba.

\section{REFERENCIAS}

AFNOR (1978). Norme Standard NF T 90-105. Détermination de matières en suspension. Association Française de Normalisation [en línea]. https://www. boutique.afnor.org/norme/nf-t90-105/essais-deseaux-determination-des-matieres-en-suspension/ article/893782/fa012821

Aubenneau M., Tahar A., Casellas C. y Wisniewski C. (2010). Membrane bioreactor for pharmaceutically active compounds removal: Effects of carbamazepine on mixed microbial communities implied in the treatment. Process Biochem. 45 (11), 1826-1831. https:// doi.org/10.1016/j.procbio.2010.04.011 
Beun J., Dircks K., Van Loosdrecht M. y Heijnen J. (2002). Poly- $\beta$-hydroxybutyrate metabolism in dynamically fed mixed microbial cultures. Water Res. 36 (5), 1167-1180. https://doi.org/10.1016/S00431354(01)00317-7

Braak E. (2012). Aération pour le décolmatage dans les bioréacteurs à membranes immergées pour le traitement des eaux usées: impact sur le milieu biologique et la filtration. Tesis de Doctorado. Universidad de Toulouse, Toulouse, Francia, 239 pp.

Buer T. y Cumin J. (2010). MBR module design and operation. Desalination 250, 1073-1077. https://doi. org/10.1016/j.desal.2009.09.111

Carta F., Beun J., van Loosdrecht M. y Heijnen J. (2001). Simultaneous storage and degradation of PHB and glycogen in activated sludge cultures. Water Res. 35 (11), 2693-2701. https://doi.org/10.1016/S00431354(00)00563-7

CEE (1991). Directive 91/271/CEE. Directive relative au traitement des eaux urbaines résiduaires. Comunidad Económica Europea [en línea]. https://eur-lex.europa. $\mathrm{eu} /$ legal-content/FR/TXT/?uri=celex\%3A31991L0271

Cicek N., Franco J.P., Suidan M.T., Urbain V. y Manem J. (1999). Characterization and comparison of a membrane bioreactor and a conventional activated-sludge system in the treatment of wastewater containing high molecular weight compounds. Water Environ. Res. 71 (1), 64-70. https://doi. org/10.2175/106143099X121481

De Luca G., Sacchetti R., Leoni E. y Zanetti F. (2013). Removal of indicator bacteriophages from municipal wastewater by a full-scale membrane bioreactor and a conventional activated sludge process: implications to water reuse. Bioresour. Technol. 129, 526-531. https:// doi.org/10.1016/j.biortech.2012.11.113

Delgado L.F., Schetrite S., Gonzalez C. y Albasi C. (2010). Effect of cytostatic drugs on microbial behaviour in membrane bioreactor system. Bioresour. Technol. 101(2), 527-536. https://doi.org/10.1016/j. biortech.2009.08.051

Di Bella G., Di Trapani D., Torregrossa M. y Viviani G. (2013). Performance of a MBR pilot plant treating high strength wastewater subject to salinity increase: analysis of biomass activity and fouling behaviour. Bioresour. Technol. 147, 614-618. https://doi.org/10.1016/j. biortech.2013.08.025

Ersahin M.E., Tao Y., Ozgun H., Spanjers H. y van Lier J.B. (2016). Characteristics and role of dynamic membrane layer in anaerobic membrane bioreactors. Biotechnol. Bioeng. 113 (4), 761-771. https://doi. org/10.1002/bit.25841

Ferrero G., Rodríguez-Roda I. y Comas J. (2012). Automatic control systems for submerged membrane bioreactors:
A state-of-the-art review. Water Res. 46, 3421-3433. https://doi.org/10.1016/j.watres.2012.03.055

Gerardi M.H. (2002). Nitrification and denitrification in the activated sludge process. John Wiley and Sons, Nueva York, EUA, 208 pp.

González-Hernández Y., Schetrite S., Albasi C., Alliet M. y Jáuregui-Haza U.J. (2019). Stoichiometry and kinetics of hospital wastewater treatment in a submerged membrane bioreactor. Desalination. Water Treat. 164, 18-30. https://doi.org/10.5004/dwt.2019.24392

Henze M. (2000). Activated sludge models ASM1, ASM2, ASM2d and ASM3. IWA Publishing, Londres, Reino Unido, $128 \mathrm{pp}$.

Hoinkis J., Deowan S.A., Panten V., Figolic A., Huang R.R. y Drioli E. (2012). Membrane Bioreactor (MBR) Technology - a Promising Approach for industrial water reuse. Procedia Eng. 33, 234-241. https://doi. org/10.1016/j.proeng.2012.01.1199

Jiménez J., Grelier P., Meinhold J. y Tazi-Pain A. (2010). Biological modelling of MBR and impact of primary sedimentation. Desalination 250, 562-567. https://doi. org/10.1016/j.desal.2009.09.024

Judd S. (2016). The status of industrial and municipal effluent treatment with membrane bioreactor technology. Chem. Eng. J. 305, 37-45. https://doi.org/10.1016/j. cej.2015.08.141

Keskes S., Hmaied F., Gannoun H., Bouallagui H., Godon J.J. y Hamdi M. (2012). Performance of a submerged membrane bioreactor for the aerobic treatment of abattoir wastewater. Bioresour. Technol. 103 (1), 28-34. https://doi.org/10.1016/j.biortech.2011.09.063

Kumar S., Groth A. y Vlacic L. (2014). An analytical index for evaluating manufacturing cost and performance of low-pressure hollow fibre membrane systems. Desalination 332, 44-51. https://doi.org/10.1016/j. desal.2013.10.013

Lin H., Wang F., Ding L., Hong H., Chen J. y Lu X. (2011). Enhanced performance of a submerged membrane bioreactor with powdered activated carbon addition for municipal secondary effluent treatment. J. Hazard. Mater. 192 (3), 1509-1514. https://doi.org/10.1016/j. jhazmat.2011.06.071

McArdell C., Kovalova L., Siegrist H., Kienle C., Moser R. y Schwartz T. (2011). Input and elimination of pharmaceuticals and disinfectants from hospital wastewater. Final project report. Eawag: Das WasserforschungsInstitut des ETH-Bereichs, Dubendorf, Suiza, $61 \mathrm{pp}$.

Nguyen T.T., Bui X.T., Luu V.P., Nguyen P.D., Guo W. y Ngo H.H. (2017). Removal of antibiotics in sponge membrane bioreactors treating hospital wastewater: Comparison between hollow fiber and flat sheet membrane systems. Bioresour. Technol. 240, 42-49. https:// doi.org/10.1016/j.biortech.2017.02.118 
Nguyen T.T., Bu X.T., Dang B.T., Ngo H.H., Jahng D., Fujioka T., Chen S.S., Dinh Q.T. y Nguyen C.N. (2019). Effect of ciprofloxacin dosages on the performance of sponge membrane bioreactor treating hospital wastewater. Bioresour. Technol. 273, 573-580. https://doi. org/10.1016/j.biortech.2018.11.058

Ouarda Y., Tiwari B., Azaïs A., Vaudreuil M.A., Ndiaye S.D., Drogui P., Tyagi R.D., Sauvé S., Desrosiers M., Buelna G. y Dubé R. (2018). Synthetic hospital wastewater treatment by coupling submerged membrane bioreactor and electrochemical advanced oxidation process: Kinetic study and toxicity assessment. Chemosphere 193, 160-169. https://doi.org/10.1016/j. chemosphere.2017.11.010

Quesada I., González Y., Schetrite S., Budzinski H., Le Menach K., Lorain O., Manier N., Ait Aissa S., Pandard P., Abdelaziz D., Canonge J.M. y Albasi C. (2015). PANACÉE: évaluation du fonctionnement d'un bioréacteur à membranes immergées traitant des effluents hospitaliers d'oncologie. J. Water Sci. 28 (1), 1-6. https://doi.org/10.7202/1030001ar

Radjenović J., Petrović M. y Barceló D. (2009). Fate and distribution of pharmaceuticals in wastewater and sewage sludge of the conventional activated sludge (CAS) and advanced membrane bioreactor (MBR) treatment. Water Res. 43 (3), 831-841. https://doi.org/10.1016/j. watres.2008.11.043

Roccaro P. y Vagliasindi F.G. (2020). Techno-economic feasibility of membrane bioreactor (MBR). En: Frontiers in water-energy-nexus-nature-based solutions, advanced technologies and best practices for environmental sustainability (Naddeo V., Balakrishnan M. y Choo K.H., Eds.). Springer, Cham, Suiza, pp. 269270. https://doi.org/10.1007/978-3-030-13068-8_67

Rudin S.N.F.M., bin Shabri H.A., Ab Muis Z., Hashim H. y Ho W.S.J.C.E.T. (2018). Value-added waste potential of wastewater sludge from pharmaceutical industry: A review. Chem. Eng. Trans. 63, 493-498. https://doi. org/0.3303/CET1863083

Shen L., Yuan X., Shen W., He N., Wang Y., Lu H. y Lu Y. (2014). Positive impact of biofilm on reducing the permeation of ampicillin through membrane for membrane bioreactor. Chemosphere 97, 34-39. https://doi. org/10.1016/j.chemosphere.2013.10.056

Sipma J., Osuna B., Collado N., Monclús H., Ferrero G., Comas J. y Rodríguez-Roda I. (2010). Comparison of removal of pharmaceuticals in MBR and activated sludge systems. Desalination 250, 653-659. https://doi. org/10.1016/j.desal.2009.06.073
Song L., Liang S. y Yuan L. (2007). Retarded transport and accumulation of soluble microbial products in a membrane bioreactor. J. Environ. Eng. 133 (1), 36-43. https:// doi.org/10.1061/(ASCE)0733-9372(2007)133:1(36)

Strotmann U., Geldem A., Kuhn A., Gendig C. y Klein S. (1999). Evaluation of a respirometric test method to determine the heterotrophic yield coefficient of activated sludge bacteria. Chemosphere 38 (15), 35553570. https://doi.org/10.1016/S0045-6535(98)00569-4 Svojitka J., Dvor L., Studer M., Straub J.O., Frömelt H. y Wintgens T. (2017). Performance of an anaerobic membrane bioreactor for pharmaceutical wastewater treatment. Bioresour. Technol. 229, 180-189. https:// doi.org/10.1016/j.biortech.2017.01.022

Tambosi J.L., de Sena R.F., Favier M., Gebhardt W., José H.J., Schröder H.F. y Moreira R.F.P.M. (2010). Removal of pharmaceutical compounds in membrane bioreactors (MBR) applying submerged membranes. Desalination 261, 148-156. https://doi.org/10.1016/j. desal.2010.05.014

Tran T., Nguyen T.B., Ho H.L., Le D.A., Lam T.D., Nguyen D.C., Hoang A.T., Do T.S., Hoang L. y Nguyen T.D.J.P. (2019). Integration of membrane bioreactor and nanofiltration for the treatment process of real hospital wastewater in Ho Chi Minh City, Vietnam. Processes 7 (3), 123. https://doi.org/10.3390/pr7030123

Vivekanandan B. y Rao A.S. (2017). Estimation of yield, growth rate, decay rate, and half-saturation coefficients of ASM1 model parameters. Int. J. Environ. Res. 11 (4), 415-423. https://doi.org/10.1007/s41742-017-0037-z

Yeole M.H.G., Lokhande M.S.A. y Mahale M.T.B. (2018). Modern outlines in membrane bioreactor for sewage treatment and industrial wastewater and their adequate growth for ZLD in India. Int. J. Eng. Technol. Sci. Res. 5 (3), 837-840.

Zanetti F., De Luca G. y Sacchetti R. (2010). Performance of a full-scale membrane bioreactor system in treating municipal wastewater for reuse purposes. Bioresour. Technol. 101, 3768-3771. https://doi.org/10.1016/j. biortech.2009.12.091

Zarragoitia-González A., Schetrite S., Jáuregui-Haza U.J. y Albasi C. (2009a). Dynamical modelling and simulation of wastewater filtration process by submerged membrane bioreactors. Int. J. Chem. React. Eng. 7 (29), 1-20. https://doi.org/10.2202/1542-6580.1856

Zarragoitia-González A. (2009b). Desarrollo de modelos dinámicos para la simulación y optimización de biorreactores con membrana sumergida para el tratamiento de aguas residuales. Tesis de Doctorado. Universidad de Toulouse, Toulouse, Francia, 129 pp. 